\title{
THE MANAGEMENT OF THE ACUTE PHASE OF POLIOMYELITIS
}

\author{
By Philip BAYeR; M.D.(Aberd.), M.R.C.P.(Lond.). \\ Physician-in-Charge, Fever Hospital, Fohannesburg. Lecturer in Infectious Diseases, University of the Witwatersrand
}

There is no disease in the field of medicine which can be more alarming not only to the patient and his relatives, but also to the doctors and staff of a hospital, than acute anterior poliomyelitis. In the period November 1947 to January 1948 a large outbreak occurred in South Africa, mostly confined to the Transvaal, and some $75^{\circ}$ cases passed through our hands at the Fever Hospital, Johannesburg.

Our first problem was the finding of sufficient bed accommodation. Other infectious diseases were therefore gradually eliminated until eventually the entire hospital was filled with poliomyelitis cases. Our next difficulty was nursing staff, already a problem of its own even in normal times. The public were naturally very frightened of this dreaded disease, particularly married nursing sisters who were afraid of taking it home to their children. It was only through an urgent appeal to the press and radio that the Johannesburg public came forward in great spirit to help us out of a serious predicament. That the risk of infection to hospital staff clearly existed was shown by the fact that six nurses, the night sisterin-charge, and one house physician contracted the disease. Additional problems also arose as the epidemic progressed, but for the moment we propose to digress somewhat from the main purpose of this paper and give a short survey of the diagnostic aspects.

The majority of cases, probably from 80 to 90 per cent., started suddenly with mild to severe evidence of meningeal irritation. Constitutional symptoms were confined to pyrexia (at times only moderate), headaches and vomiting. Diarrhoea, sore throat, and joint or muscular pains were not prominent features, at least not in the early stages. Clinical examination in this phase could be very misleading and signs had to be looked for most carefully. Stiffness of the neck and head retraction were at times indefinite and Kernig's sign debatable, whilst other evidence of neurological involvement was frequently singularly negative. However there remained one definite positive feature, namely stiffness of the back. In some cases this was so marked as to be absolutely diagnostic provided, of course, that local causes of a stiff back had been excluded. There remained, however, a group of cases where the question of a stiff back was somewhat indefinite and could possibly be due to the natural physical state of the suspected patient. To demonstrate this stiffness the patient was asked to sit up, and with the knees slightly flexed, place his head between his knees. It is obvious that the middle-aged obese female was at a disadvantage, whereas the athletic type could disguise somewhat any spasm of the back muscles. In view of what has been said, at first the local practitioners were not prepared to diagnose poliomyelitis in this type of case, and often waited until some days later when paralytic phenomena appeared. But it was just this very mild type of case ('Poker-back polio' became the colloquial term) that often aborted completely and showed no residua, and positive diagnosis could only be established by lumbar puncture. Here again further difficulties arose. In approximately Io per cent. of patients suffering from poliomyelitis the cerebro-spinal fluid may remain normal throughout. Furthermore the criteria of what represents a normal cerebro-spinal fluid varies with different authorities. A bloody puncture often completely spoilt the value of this diagnostic procedure. Even a few polymorphonuclear cells were regarded with suspicion, especially when associated with a total protein content of over $50 \mathrm{mg}$. per cent. Later on in the epidemic it became necessary to re-lumbar puncture certain doubtful cases and every now and then positive findings occurred establishing the diagnosis. For quite a time one was personally very dubious in diagnosing poliomyelitis in a subject with slight or doubtful stiff back and a normal cerebro-spinal fluid. But as time went on it was clear that the great majority of these cases were suffering from true poliomyelitis. Our reason for this was that it was surely more than a coincidence that the same type of picture was being seen every day in large numbers. What other mysterious disease could be epidemic at the time if not poliomyelitis? $\mathrm{Re}$ peated lumbar punctures and the subsequent development of residua proved beyond all doubt that 
the disease was poliomyelitis. It is quite likely that certain cases of hysteria, not uncommon at the terminal stage of the epidemic, fibrositis, etc., were treated as poliomyelitis, but one was satisfied that the bulk of these 'stiff back' cases were suffering from poliomyelitis.

These mild cases were a veritable nightmare to the admission staff of the Fever Hospital. There were not enough cubicle isolation beds to deal with the ever increasing numbers, increasing too because the signs and symptoms of poliomyelitis were frequently described in the press, and doctors and public alike began to regard every febrile illness as poliomyelitis. It was essential not to bring these suspects into contact with established cases, so two beds were put into every single cubicle. By doubling the cubicle capacity of the hospital it was thus possible to give some sort of isolation to the suspected cases. Immediately on admission, suspects were examined with meticulous care especially for the presence or absence of a 'stiff back.' This sign in the abortive cases may last only a day or two, and if not detected immediately on admission the ideal time for diagnosis is lost, and a final diagnosis may never be possible in that particular case. Lumbar puncture was performed as a routine but was not repeated except in certain selected cases for diagnostic purposes.

With regard to the nursing of the patient the general principles applied in the treatment of any febrile disease were adopted. To prevent sagging of the bed, fracture boards were inserted, and another board was most useful for supporting the feet and preventing foot drop. A most careful check was kept on the pulse and respiration rate, on the urinary function and on the patient's ability to swallow. As the muscles are so very painful in the early stages of the disease the patients were handled as little as possible and given the greatest possible rest. All patients were put under an electric cradle made out of wire about $5 \mathrm{ft}$. long and heated by means of six to eight electric globes. There is no doubt that heat applied in this way was most soothing and could be kept up day and night for many weeks if necessary. There was not sufficient nursing staff to give every patient hot packs, a most effective remedy for relieving severe spasm, but in severe cases, packs were applied every few hours and found most useful. An appreciable proportion of patients developed retention of urine. A constant check was therefore kept on the urinary output and signs of a distended bladder were sought. Where a hot pack applied to the bladder failed, catheterization was resorted to, and in severe cases a catheter had to be tied in for many weeks until normal urinary function was established.
Painful spasms were encountered very frequently and required a good deal of attention to relieve suffering. Heat applied in the manner mentioned above was of great help in relieving spasm, but at times failed in severe cases. Curare was not used as its use had not yet been sufficiently standardized, and there had been too many unfortunate accidents reported as the result of its injudicious use. One of our anaesthetist colleagues gave continuous intravenous infusions of procaine to a small group of severe cases. The patients had to be watched day and night, veins were always giving trouble, and one saw such little relief of spasm that this form of therapy, with all the trouble it entailed, was hardly worth being given a more extensive trial. Prostigmin was often employed with very doubtful results. Etamon was more effective, but it is a toxic drug, and must be used with care.

It has been suggested that the paretic effect of poliomyelitis may in part be due to anoxaemia of nerve cells, rather than to the lethal action of the virus. This might be overcome to some extent by the use of continuous oxygen, in spite of the fact that the oxygen saturation level of the blood can be raised only very slightly by oxygen inhalation. Consequently every patient immediately on admission was put on continuous oxygen by nasal catheter. This form of treatment was not difficult to carry out except in young children. Unfortunately, oxygen therapy was started in the latter half of the epidemic and therefore it is difficult to give a true picture of its value in treatment, particularly with regard to its effect on paralytic phenomena. The hospital staff, however, were emphatic that patients appeared to be more comfortable and the frequent use of sedatives was no longer necessary. In a few cases a complete oxygen tent was employed. It was irksome and frightening to some patients, however, and the apparatus is complicated and requires constant attention. It was not persevered with. One feels that this form of therapy should be given a more extensive trial. A most careful watch was always kept on the development of paralysis, particularly where this appeared to spread.

Unfortunately an erroneous impression is given by the older textbooks of medicine, that the paralysis once established is fixed and thereafter either remains static or improves. Not infrequently a slight paresis was seen, days or even weeks later, to progress to a severe palsy involving many more muscles than were at first affected. It is most important to remember this aspect from the prognostic point of view, and although one does not want to cause panic, it is better and far wiser to give a guarded prognosis in the early stages of the disease. 
As has been stated already, for the first ten days the patients were disturbed as little as possible and examined only when necessary. The usual principle of placing limbs in the position of greatest usefulness afterwards was adopted. Thus the arms were kept abducted by means of a pad of wool and sandbags, and the wrists cocked up to prevent wrist drop. In the great majority of cases the deltoids are affected in the upper limb paralysis, and so in children particularly, the arms were extended above the head and pinned on to a cushion at varying intervals during the day. In the lower extremities the limbs were slightly abducted, bent at the knee and the ankles dorsiflexed by an adjustable board at the foot of the bed. Sandbags were used to maintain position, not splints. At frequent intervals, the position of limbs was changed to prevent undue strain on muscles and to ease the patient's discomfort. If after ten days the febrile stage had subsided and the general condition was satisfactory, the physiotherapist was introduced for the first time. A careful survey of the affected muscles was first undertaken and carefully recorded. Unless muscle spasm was severe mild massage and active and passive movements were instituted, and splinting was adopted where necessary. One understands that the value of this form of therapy has been the subject of a great deal of controversy in the profession, but it is certain that it has a most beneficial effect upon the patient's psychological outlook, and for that reason should always be used. The patient feels that at last something is being done for him and that he is beginning to recover. Even in young children once the physiotherapist has won their confidence a much brighter and more useful outlook becomes manifest.

The value of drugs is very limited in treatment. So far no specific remedies have been found and chemo-therapy, penicillin and streptomycin, as well as sulphonamides have been found ineffectual. At this stage it should be mentioned that occasionally meningitis, particularly meningococcal meningitis, closely resembles the meningitic form of poliomyelitis in the pre-paralytic stage. Meningococci are sometimes difficult to find in the blood or cerebro-spinal fluid, especially as practitioners so often use penicillin and sulphonamides early in any febrile disease. By the time lumbar puncture is performed organisms cannot be found. The cerebro-spinal fluid findings, namely, increased protein with polymorph and lymphocytic cells, and a normal sugar reading may be indistinguishable from the findings in many cases of poliomyelitis. For this reason it is strongly advisable to use chemo-therapy in all doubtful cases until diagnosis is fully established. This could do no possible harm to poliomyelitis but might prove life saving in meningitis. For the respiratory complications of poliomyelitis there is of course no objection whatsoever to the free use of penicillin and sulphonamides in the hope that they may prevent the spread of the infection. For pain, simple sedatives and analgesics were employed wherever necessary, whilst in adults luminal was effective in allaying the terrible anxiety so often experienced by intelligent patients who realized only too clearly their plight.

\section{Bulbar Palsy}

This is the most serious complication of poliomyelitis and is responsible for most deaths. It may come on with alarming rapidity, and for this reason it is always advisable to admit cases of poliomyelitis as early as possible to hospital. Bulbar paralysis may show itself in two main forms, depending upon whether the cardiac or the respiratory centre is affected. Often both are involved or there is sufficient overlapping to prevent a true differentiation. A failing cardiac centre is revealed by a thready, rapid, regular pulse with falling blood pressure. When the respiratory centre fails, the patient's breathing becomes irregular and shallow, and cyanosis develops, associated with intense anxiety and sweating.

Not infrequently there is in addition involvement of the nuclei of the ninth to the twelfth nerves, giving rise to such symptoms as weakness of the soft palate, regurgitation of fluids through the nose and slurring of speech; furthermore there may be difficulty in coughing and swallowing. This combined lack of swallowing and coughing allows mucus and saliva to accumulate in the pharynx, greatly impeding respiration and leading to aspiration pneumonia. It is obvious that this type of case requires very special care, constant observation and skilful nursing.

Firstly the foot of the bed is raised and the patient placed on his side to obtain postural drainage. Next, almost continuous suction through an aspirator is instituted. It is now advocated by many leading American authorities that in certain cases a tracheotomy may prove a life saving measure as it greatly facilitiates respiration. Continuous oxygen is of course essential and as the patient cannot swallow, fluid is given by the intravenous drip method. Usually 5 to 10 per cent. glucose in sterile water or saline is used. It cannot be sufficiently emphasized that great care should be taken in the administration of intravenous fluid because if too much is given, pulmonary oedema, with fatal results, is likely to ensue.

To maintain the patient's strength some nourishment is essential. This is done by in- 
serting a nasal catheter through the nose into the stomach and feeding the patient through the tube. The trained sister of a fever hospital soon becomes highly expert at passing the catheter into the oesophagus and not into the bronchus, and fluid nourishment in the form of milk, egg flips, etc., can be given very conveniently. The tube need not be taken out daily unless it gets blocked, but it should not be left in too long as it undoubtedly causes discomfort. Great care should be taken when feeding by the mouth is again instituted and it is wise to start very slowly with sips of water, broth and substances such as jelly.

With or without evidence of bulbar paralysis there may be great difficulty in breathing, owing to paralysis of the intercostal muscles and diaphragm. In upper. arm paralysis, particularly when the deltoids are involved, careful watch must be kept for the development of diaphragmatic paralysis. Clinically the condition can be easily recognized, but it is well to reme nber that it can come on. dramatically and it is wise to be prepared for such an emergency by having an artificial respirator such as the Nuffield type of iron lung in readiness. The Nuffield lung was found most satisfactory, but during the war the South African Air Force constructed a number of iron lungs with certain innovations which facilitated treatment. The entire lung was constructed of metal, obviating the leaks occasionally developing in the Nuffield lung, and more vents capable of being opened and closed easily were put in, making possible easier access to the patient without actually removing him from the lung.

There appears to be a great difference of opinion as to which type of case should be placed in the iron lung. To begin with it is stated that bulbar cases do badly in the lung and should be kept out whenever possible. This certainly has not been our experience here. It is difficult to see why an iron lung should have a bad effect on this type of case. For all other forms of treatment to be carried out whilst the patient is to some extent relieved of the problem of breathing is surely an advantage. It is our view that bulbar cases do badly not because of the iron lung but because of the disease itself. Two serious objections to the iron lung are that it does in the beginning have a bad psychological effect, amounting at times to panic in older patients, and as each case has to be 'specialled' the strain on the nursing staff is aggravated. One can appreciate the difficulty of nursing a patient in the lung with a tube in the leg for intravenous medication, one through a nostril for oxygen, another for nasal feeding, a mouth tube for suction and a urethral catheter for retention of urine. Another objection to the use of the iron lung is that paralysis cannot be given proper treatment; but at this stage with the patient's life so gravely threatened, the little good that can be expected from physiotherapy is not really important. It has also been suggested that complications such as atelectasis are more common after the use of a respirator. Philip Stimson suggests that bulbar cases where there is great difficulty in swallowing should first have a tracheotomy performed before being placed in the lung. The object of tracheotomy is to enable repeated suction to be carried out and to keep the trachea clear of all mucus, so that oxygen easily reaches the lungs.

As the patient recovers there comes a time usually after two to three weeks, when an attempt is made to get him to breathe naturally. It is essential to go very slowly at first. Although the respiratory centre may have recovered sufficiently for natural breathing, often after several days it appears to weaken and symptoms recur, necessitating a return to the lung. At times it may take many weeks and even months of graduated training until the patient can discard the iron lung altogether. Abortive cases, especially those without residua, are allowed up at the end of three weeks, but are given a most thorough examination to detect any latent defects. If none are found they are allowed to go home but advised to avoid strenuous exercise for several months. Unfortunately a small percentage had a recurrence of the disease and had to be re-admitted for treatment.

The mortality in this epidemic was approximately ro per cent., mostly due to bulbar involvement. At least 50 per cent. were abortive cases and recovered completely. Of the remaining 40 per cent. about 25 per cent. were only slightly affected, but in 15 per cent. the paralysis was severe and was likely to cause permanent crippling. All cases with sequelae after three to four weeks of treatment were transferred to a special ward set aside at the General Hospital, Johannesburg. Here they came into the charge of physiotherapists assisted by a team of orthopaedic surgeons to deal with the many disabling effects of a disease which at the time of writing still baffles us as regards its epidemiology, its mode of transmission, and its effective treatment. 